\title{
Virulence Phenotypes and Molecular Genotypes in Collections of Puccinia triticina from Italy
}

Paola Mantovani, Marco Maccaferri, and Roberto Tuberosa, Department of Agroenvironmental Sciences and Technology, Viale Fanin 44, 40127, Bologna, Italy; and James Kolmer, Cereal Disease Laboratory, Agricultural Research Service, U.S. Department of Agriculture, 1551 Lindig Avenue, St. Paul, MN 55108, USA

\begin{abstract}
Mantovani, P., Maccaferri, M., Tuberosa, R., and Kolmer, J. 2010. Virulence phenotypes and molecular genotypes in collections of Puccinia triticina from Italy. Plant Dis. 94:420-424.

Twenty-four isolates of Puccinia triticina from Italy were characterized for virulence to seedlings of 22 common wheat Thatcher isolines, each with a different leaf rust resistance gene, and for molecular genotypes at 15 simple sequence repeat (SSR) loci. The isolates were compared to a set of 13 previously characterized $P$. triticina isolates from either durum or common wheat. Clustering based on virulence phenotypes and SSR genotypes grouped the Italian P. triticina isolates into three groups. In the first group, the isolates had virulence phenotypes and SSR genotypes that were similar to the isolates collected from durum wheat. Isolates in the second group were unique because they had virulence similar to the isolates from common wheat but were distinct for SSR genotypes compared to the isolates from durum wheat and from common wheat. Isolates in the third group had virulence phenotypes and SSR genotypes closely related to the isolates from common wheat. The isolates were grouped based on the known or assumed host of origin, virulence phenotype, and SSR genotypes. Measures of $F_{\mathrm{ST}}$ and $R_{\mathrm{ST}}$ for SSR genotypes, and $\Phi_{\mathrm{ST}}$ for virulence phenotype were significant, which indicated differentiation among the three groups of isolates. Virulence phenotypes and molecular genotypes were highly correlated with $r=0.74$.
\end{abstract}

Durum wheat (Triticum turgidum L.) and common wheat (T. aestivum L.) are both widely cultivated in Italy. Tetraploid wheats (emmer and durum types with $\mathrm{AB}$ genomes) have been cultivated in the Middle East and Mediterranean region for thousands of years. Cultivated durum wheat first appeared in the Mediterranean region as a major crop during the Greek civilization, ca. 2,300 years ago (2). Leaf rust caused by Puccinia triticina Eriks. is a very common disease of both durum and common wheat, with distinct races or virulence phenotypes of $P$. triticina found on durum and common wheat $(18,23)$. The $P$. triticina isolates collected from durum wheat are avirulent to many of the leaf rust resistance genes present in common wheat. $P$. triticina isolates collected from common wheat are more variable for virulence compared to the collections from durum wheat $(4,7)$. In addition, the collections of $P$. triticina from durum wheat had distinct simple sequence repeat (SSR) genotypes compared to all of the major groups of $P$.

Corresponding author: James Kolmer

E-mail: JKolmer@umn.edu

Accepted for publication 8 December 2009.

doi:10.1094/PDIS-94-4-0420

This article is in the public domain and not copyrightable. It may be freely reprinted with customary crediting of the source. The American Phytopathological Society, 2010. triticina collected from common wheat $(9,18)$.

The discrete separation between the $P$. triticina isolates collected from durum and common wheat suggested a highly divergent selection of $P$. triticina driven by host genotype. However, some variations in the isolates from durum wheat have been noted. In Ethiopia, which is considered to be a secondary center of diversity of tetraploid wheats (20), isolates of $P$. triticina from durum wheat were distinct for SSR genotype compared to isolates collected from cultivated durum wheat in Europe, South America, and Mexico (18).

The genetics of leaf rust resistance in durum wheat has recently been examined $(5,6)$ due to the emergence of a new virulence phenotype of $P$. triticina that was virulent to many durum cultivars worldwide (23). In order to find new sources of leaf rust resistance in durum wheat, genetic analysis should be conducted with $P$. triticina isolates specialized to this host species, since the virulence of the isolates can influence the results and conclusions of these studies (12).

In Italy, durum wheat is an important crop; however, little is known about the virulence of the $P$. triticina isolates collected in this country. A set of $P$. triticina isolates were collected from various resistant and susceptible durum cultivars in different locations in Italy from 1995 to 2006. Since these isolates had not been characterized for virulence, it was possible that some of them had virulence phenotypes similar to isolates from common wheat, as isolates with characteristics of those from common wheat can be collected from susceptible durum cultivars (19). A second set of isolates from Italy was previously characterized for virulence and for random amplified polymorphic DNA (RAPD) phenotypes as part of a larger study (8). However, the host origin of these isolates was not known.

The objectives of the current study were to characterize virulence and SSR genotypes of these two collections of Italian $P$. triticina isolates in order to determine their host specialization and to properly utilize them in genetic analysis for leaf rust resistance genes. We wanted to determine if these two sets of $P$. triticina isolates had virulence and molecular genotypes similar to those of other isolates collected from durum wheat, or if some of these isolates had characteristics of isolates collected from common wheat. To this purpose, the Italian isolates were analyzed in comparison to a small set of European, Mexican, and Ethiopian isolates of known host origin, virulence, and molecular genotype.

\section{MATERIALS AND METHODS}

$P$. triticina isolates. Fifteen Italian $P$. triticina isolates were collected from durum wheat cultivars grown in Italy from 1995 to 2006, and increased on the durum wheat cultivars from which they were collected. These isolates were provided by the seed company Produttori Sementi Bologna (PSB; Argelato, BO, Italy) and designated as PSB isolates. Nine Italian isolates of unknown host origin collected in 1994 (provided by F. Cassulli) were also tested for virulence phenotypes and SSR genotypes and were coded as ITA isolates. The analysis also included a set of reference isolates composed of (i) eight isolates collected from common wheat in France previously characterized for virulence phenotype (provided by H. Goyeau, INRA Grignon, France), and (ii) five isolates representative of $P$. triticina collected from durum wheat in France, Spain, Ethiopia, and Mexico (18). For virulence and molecular analysis, single-uredinial isolates were developed following standard procedures (19). Host origin, country of origin, and year of collection for the $P$. triticina isolates are listed in Table 1. 
Virulence phenotype determination. The $P$. triticina isolates were tested for virulent/avirulent infection type on seedlings of 22 near-isogenic lines of the common wheat $\mathrm{cv}$. Thatcher, each carrying one of the following leaf rust resistance genes: $\operatorname{LrB}$ (isogenic line RL6047), $\mathrm{Lrl}$ (RL6003), Lr2a (RL6000), Lr2c (RL6047), Lr3a (RL6002), Lr3bg (RL6042), Lr3ka (RL6007), Lr9 (RL6010), Lr10 (RL6004), Lr11 (RL6053), Lr14a (RL6013), Lr14b (RL6006), Lr16 (RL6005), Lr17 (RL6008), Lr18 (RL6009), Lr20 (RL6092), Lr23 (RL6012), Lr24 (RL6064), Lr26 (RL6078), Lr28 (RL6079), Lr30 (RL6049), and Lr33 (RL6057). Seedlings of Thatcher were included in all tests as a susceptible control. Inoculation, incubation, and greenhouse conditions were as previously described (19). Seedlings were scored for infection types 10 to 12 days after inoculation using the 0 to 4 scale described by Long and Kolmer (11). Infection types 0 to $2^{+}$were considered avirulent, and infection types 3 to 4 were considered virulent. Virulence phenotypes of the P. triticina isolates were described with the three-letter code used for leaf rust race nomenclature (11), plus a fourth letter based on infection type to lines with genes $\operatorname{LrB}, \operatorname{Lr} 10, \operatorname{Lr} 14 a$, and $L r 18$, and a fifth letter based on infection type to lines with genes $\mathrm{Lr} 3 \mathrm{bg}, \mathrm{Lr} 14 \mathrm{~b}$, $L r 20$, and $L r 28$. Thatcher lines with $L r 23$ and $L r 33$ were included in the differential set because these were postulated to have been derived from durum wheat (16) and because virulence to these genes was found in $P$. triticina isolates from durum wheat $(7,19)$. Virulence of isolates to $L r 23$ and $L r 33$ was not included in the five-letter race nomenclature.

Molecular genotype determination. $P$. triticina isolates were profiled with 15 SSR primer pairs specifically developed for genetic analysis of $P$. triticina: $R B 8$ and RB11 (1); and Pt61, Pt68-1, Pt91, Pt92, Pt138, Pt152, Pt154, Pt158, Pt161, Pt164, Pt173, Pt184, and Pt186 (25). DNA was extracted from 20 to $30 \mathrm{mg}$ of urediniospores that were ground by shaking with $25 \mathrm{mg}$ of sterile diatomaceous earth for 20 $\mathrm{s}$ in a Savant FastPrep shaker (FP120; Holbrook, New York). The Omniprep DNA genomic extraction kit (GenoTech, St. Louis) was used to obtain $P$. triticina DNA. After extraction, DNA was quantified with a NanoDrop ND 100 spectrophotometer (Wilmington, DE), diluted to a final concentration of $5 \mathrm{ng}^{-1} \mathrm{l}^{-1}$, and stored at $4^{\circ} \mathrm{C}$. P. triticina DNA was amplified according to the previously described protocol (18) using 5'-labeled fluorescent forward SSR primers (IR700; LI-COR, Lincoln, NE, USA). Amplification products were separated on a $7 \%$ polyacrylamide gel and visualized on a 4200 Gene Read IR Automated Genotyper with SAGA software (LI-COR). SSR allele sizes were determined using an IRDye 700 size standard (50 to $700 \mathrm{bp}$; LI-COR).
Data analysis. The 0 to 4 scale used for infection types was converted into a binary code of 1 and 0 that corresponded to virulence or avirulence of the isolate to a differential line, respectively. A matrix of number of virulence differences between each pair of isolates was derived with GenAlEx 6 (21) using the option Distance - Genetic - Binary (Haploid). Neighborjoining trees (1,001 in total) based on the virulence difference matrix were derived with PowerMarker 3.25 (10), and bootstrap values were obtained with the CONSENSE program in Phylip 3.67 (3). The matrix of virulence differences between isolates was plotted in a two-dimensional principal coordinate graph with GenAlEx 6 . The level of differentiation for virulence phenotypes between the groups of isolates was computed in GenAlEx 6 with $\Phi_{\mathrm{PT}}$ with 999 permutations of the dataset.

A matrix of SSR allele differences between all pairs of isolates was derived with GenAlEx 6. Neighbor-joining trees $(1,001$ in total) based on the SSR distance matrix were obtained with PowerMarker 3.25, and bootstrap values were obtained with the CONSENSE program in Phylip 3.67. Estimation of genetic differentiation between groups of isolates based on SSR genotypes was obtained with $F_{\mathrm{ST}}(17)$ that assumes an infinite alleles model, and $R_{\mathrm{ST}}(24)$ that assumes the stepwise mutation model. $F_{\mathrm{ST}}$ and $R_{\mathrm{ST}}$ values were calculated in GenAlEx 6 with 999 permutations of the dataset. A similarity matrix based on the number of SSR allele differences between pairs of $P$. triticina isolates was calculated with the Dice coefficient in NTSYS-pc v. 2.1 (Exeter Software, Setauket, NY). The relationship between the SSR genotypes and virulence phenotypes of the P. triticina isolates was determined by correlation analysis between the matrix of Dice coefficients for SSR genotypes and the matrix of simple matching coefficients derived from the virulence phenotypes, using the MXCOMP subprogram in NTSYS-pc v. 2.1 with 1,000 permutations of the datasets.

\section{RESULTS}

Among the 15 PSB isolates, there were eight virulence phenotypes identified with the 22 Thatcher isogenic lines (Table 2). Seven PSB isolates (8-1, 16-2, 9-3, 10-3, 1-3, 6-2, and 14-3) had virulence phenotypes of BBBGG (virulent to $L r 10, L r 14 b$, and $L r 33$ ), BBBQG (virulent to $\operatorname{LrB}, \operatorname{Lr} 10$, $L r 14 b$, and $L r 33$ ), or BBBGJ (virulent to $L r 10, L r 14 b, L r 20, L r 23$, and Lr33), and isolate 11-3 had a DCBQG phenotype,

Table 1. Host origin, country of origin, and year of collection of 37 Puccinia triticina isolates collected from common and durum wheat

\begin{tabular}{|c|c|c|c|}
\hline Isolate & Country & Host origin & Year of collection \\
\hline PSB 1-3 & Italy & Durum wheat & 1995 \\
\hline PSB 2-2 & Italy & Durum wheat & 1995 \\
\hline PSB 3-3 & Italy & Durum wheat & 1995 \\
\hline PSB 4-2 & Italy & Durum wheat & 2004 \\
\hline PSB 5-2 & Italy & Durum wheat & 1995 \\
\hline PSB 6-2 & Italy & Durum wheat & 1995 \\
\hline PSB 7-3 & Italy & Durum wheat & 1995 \\
\hline PSB 8-1 & Italy & Durum wheat & 1997 \\
\hline PSB 9-3 & Italy & Durum wheat & 1995 \\
\hline PSB 10-3 & Italy & Durum wheat & 2003 \\
\hline PSB 11-3 & Italy & Durum wheat & 2006 \\
\hline PSB 13-2 & Italy & Durum wheat & 2006 \\
\hline PSB 14-3 & Italy & Durum wheat & 2004 \\
\hline PSB $15-1$ & Italy & Durum wheat & 2006 \\
\hline PSB 16-2 & Italy & Durum wheat & 2006 \\
\hline ITA 1-1 & Italy & Unknown & 1994 \\
\hline ITA $1-2$ & Italy & Unknown & 1994 \\
\hline ITA 2-1 & Italy & Unknown & 1994 \\
\hline ITA 2-2 & Italy & Unknown & 1994 \\
\hline ITA 4-1 & Italy & Unknown & 1994 \\
\hline ITA 7-1 & Italy & Unknown & 1994 \\
\hline ITA $12-2$ & Italy & Unknown & 1994 \\
\hline ITA 14-1 & Italy & Unknown & 1994 \\
\hline ITA $15-1$ & Italy & Unknown & 1994 \\
\hline F 57 & France & Common wheat & 2004 \\
\hline F 59 & France & Common wheat & 2004 \\
\hline F 60 & France & Common wheat & 2004 \\
\hline F 61 & France & Common wheat & 2004 \\
\hline F 62 & France & Common wheat & 2004 \\
\hline F58 & France & Common wheat & 2004 \\
\hline F 64 & France & Common wheat & 2004 \\
\hline F 66 & France & Common wheat & 2004 \\
\hline FS 3.1 & France & Durum wheat & 2003 \\
\hline Esp 22 & Spain & Durum wheat & 2003 \\
\hline Eth 6.1-1 & Ethiopia & Durum wheat & 2002 \\
\hline Eth 7.2 & Ethiopia & Durum wheat & 2002 \\
\hline MX 14.3 & Mexico & Durum wheat & 2002 \\
\hline
\end{tabular}


with virulence to $L r 2 c, L r 26, L r B, L r 10$, $L r 14 b$, and $L r 33$. All of these isolates were virulent to $L r 10$ and $L r 14 b$. These eight PSB isolates were avirulent to most of the Thatcher near-isogenic lines and were very similar for virulence to isolates FS 3.1, Esp 22, Eth 7.2, and MX 14.3 that were collected from durum wheat in France, Spain, Ethiopia, and Mexico, respectively. Isolate Eth 6.1-1 from Ethiopia had low infection type to Thatcher, and therefore had low infection to all the Thatcher isogenic lines. The other seven PSB isolates (2-2, 3-3, 4$2,5-2,7-3,15-1$, and 13-2) had virulence phenotypes of FGBQQ with virulence to Lr2c, Lr3, Lr16, LrB, Lr10, Lr3bg, Lr14b, and $L r 33$; FGBQS with virulence to $L r 2 c$, Lr3, Lr16, LrB, Lr10, Lr3bg, Lr14b, Lr20, and $L r 33$; and FBBQQ with virulence to Lr2c, Lr3, LrB, Lr10, Lr3bg, LrI4b, and $L r 33$. Of the nine ITA isolates collected in Italy in 1994, six (1-1, 1-2, 2-1, 2-2, 15-1, and 7-1) had virulence phenotypes of BBBGG (virulent to $L r 10, L r 14 b$, and Lr33), BBBGK (virulent to Lr10, Lr14b, Lr20, Lr28, Lr23, and Lr33), and DBBGJ (virulent to $L r 2 c, L r 10, L r 14 b, L r 20, L r 23$, and Lr33) that were also similar to the isolates collected from durum wheat in France, Spain, Ethiopia, and Mexico. The other three ITA isolates (4-1, 12-2, and 141) had FCTSQ, FGPSQ, and FGMNS virulence phenotypes, similar to the French isolates that were collected from common wheat.

In the neighbor-joining dendrogram of virulence differences between isolates in Figure 1, three groups of isolates could be distinguished. Group 1 consisted of eight PSB isolates, six ITA isolates, and the five isolates collected from durum wheat in France, Spain, Ethiopia, and Mexico. These isolates all had virulence phenotypes of B---- or D----, characteristic of isolates from durum wheat. Group 2 consisted of the seven PSB isolates with F---virulence phenotypes that were also virulent to $\operatorname{Lr16}, \mathrm{LrB}, \mathrm{Lr} 10, \mathrm{Lr} 3 \mathrm{bg}$, and $\operatorname{Lrl} 14 b$. Isolates in Group 2 had virulence phenotypes more typical of collections from common wheat than from durum wheat. Group 3 consisted of six isolates from

Table 2. Virulence phenotypes of Puccinia triticina isolates tested for infection type to 22 Thatcher differential lines

\begin{tabular}{|c|c|c|c|}
\hline Isolate & Virulences & $\begin{array}{c}\text { Virulence } \\
\text { phenotype }\end{array}$ & $\begin{array}{c}\text { Genetic } \\
\text { differentiation } \\
\text { group }^{\mathrm{a}}\end{array}$ \\
\hline PSB 1-3 & $10,14 b, 20,33$ & BBBGJ $^{b}$ & 1 \\
\hline PSB 6-2 & $10,14 b, 20,23,33$ & BBBGJ & 1 \\
\hline PSB 8-1 & $10,14 b, 23,33$ & BBBGG & 1 \\
\hline PSB 9-3 & $10,14 b, 20,23,33$ & BBBGJ & 1 \\
\hline PSB $10-3$ & $10,14 b, 20,23,33$ & BBBGJ & 1 \\
\hline PSB 11-3 & $2 c, 26, B, 10,14 b, 33$ & DCBQG & 1 \\
\hline PSB 14-3 & $10,14 b, 20,23,33$ & BBBGJ & 1 \\
\hline PSB $16-2$ & $B, 10,14 b, 33$ & BBBQG & 1 \\
\hline PSB 2-2 & $2 c, 3 a, 16, B, 10,3 b g, 14 b, 20,33$ & FGBQS & 2 \\
\hline PSB 3-3 & $2 c, 3 a, 16, B, 10,3 b g, 14 b, 33$ & FGBQQ & 2 \\
\hline PSB 4-2 & $2 c, 3 a, 16, B, 10,3 b g, 14 b, 33$ & FGBQQ & 2 \\
\hline PSB 5-2 & $2 c, 3 a, 16, B, 10,3 b g, 14 b, 33$ & FGBQQ & 2 \\
\hline PSB 7-3 & $2 c, 3 a, 16, B, 10,3 b g, 14 b, 33$ & FGBQQ & 2 \\
\hline PSB 13-2 & $2 c, 3 a, B, 10,3 b g, 14 b, 33$ & FBBQQ & 2 \\
\hline PSB $15-1$ & $2 c, 3 a, 16, B, 10,3 b g, 14 b, 33$ & FGBQQ & 2 \\
\hline ITA $1-1$ & $10,14 b, 23,33$ & BBBGG & 1 \\
\hline ITA $1-2$ & $2 c, 10,14 b, 20,23,33$ & DBBGJ & 1 \\
\hline ITA 2-1 & $2 c, 10,14 b, 20,23,33$ & DBBGJ & 1 \\
\hline ITA 2-2 & $2 c, 10,14 b, 20,23,33$ & DBBGJ & 1 \\
\hline ITA $7-1$ & $10,14 b, 20,28,23,33$ & BBBGK & 1 \\
\hline ITA $15-1$ & $2 c, 10,14 b, 20,23,33$ & DBBGJ & 1 \\
\hline ITA 4-1 & $2 c, 3 a, 26,3 k a, 11,17,30, B, 10,14 a, 3 b g, 14 b, 33$ & FCTSQ & 3 \\
\hline ITA $12-2$ & $2 c, 3 a, 16,3 k a, 17,30, B, 10,14 a, 3 b g, 14 b, 33$ & FGPSQ & 3 \\
\hline ITA $14-1$ & $2 c, 3 a, 16,3 k a, 30, B, 14 a, 3 b g, 14 b, 20,23,33$ & FGMNS & 3 \\
\hline F 57 & $1,3 a, 17, B, 10,14 a, 3 b g, 14 b, 20,33$ & MBDSS & 3 \\
\hline F 58 & $1,2 c, 11, B, 10,14 b$ & NBGQG & 3 \\
\hline F 59 & $2 c, 3,26,3 \mathrm{ka}, 17,30, \mathrm{~B}, 10,14 a, 3 \mathrm{bg}, 14 \mathrm{~b}, 20$ & FCPSS & 3 \\
\hline F 60 & $2 c, 16,11, B, 10,14 b$ & DGGQG & 3 \\
\hline F 61 & $2 c, 3 a, 26,3 k a, 17,30, B, 10,14 a, 3 b g, 14 b, 33$ & FCPSQ & 3 \\
\hline F 62 & $1,3 a, 26,17, B, 10,14 a, 3 b g, 14 b, 20,33$ & MCDSS & 3 \\
\hline F 64 & $2 c, 3 a, 26,3 k a, 17,30, B, 14 a, 3 b g, 14 b, 33$ & FCDSS & 3 \\
\hline F 66 & $2 c, 3 a, 3 k a, 30, B, 10,14 a, 18,3 b g, 14 b, 33$ & FBMTQ & 3 \\
\hline FS 3.1 & $B, 10,14 b, 20,23,33$ & BBBQJ & 1 \\
\hline Esp 22 & $2 c, B, 10,14 b, 20,23,33$ & DBBQJ & 1 \\
\hline Eth 6.1-1 & c & BBBBB & 1 \\
\hline Eth 7.2 & $B, 14 a, 18,14 b, 20,33$ & BBBPJ & 1 \\
\hline MX 14.3 & $B, 10,14 b, 20,23,33$ & BBBQJ & 1 \\
\hline
\end{tabular}

${ }^{\text {a }}$ Groups based on host origin, virulence phenotype, and simple sequence repeat genotypes.

${ }^{\mathrm{b}}$ Five-letter code describing virulence phenotypes to 20 isogenic lines adapted from Long and Kolmer (11) and Ordoñez and Kolmer (19). Virulence to Lr23 and Lr33 not included in five-letter code.

c Avirulent to all the seedling $L r$ genes tested.
France and three ITA isolates with F---and M---- virulence phenotypes that were characteristic of isolates from common wheat. Two isolates from France collected from common wheat, F58 and F60, had NBGQQ and DCGQG virulence phenotypes, respectively, and clustered most closely with isolates in Group 1. Principal coordinate analysis in Figure 2 grouped the isolates in the same manner as the neighbor-joining dendrogram. The first principal coordinates (Pcol) and the second principal coordinates (Pco2) accounted for 50.4 and $18.5 \%$ of the total variation, respectively. Pco1 distinguished the isolates from common wheat (Fig. 1Group 3) clustered on the left side of the diagram from the isolates from durum wheat (Fig. 1-Group 1) that were on the right side of the plot. The seven PSB isolates had three virulence phenotypes similar to common wheat isolates (Fig. 1Group 2) that were in the lower part of the plot along with isolates F58 and F60 from common wheat.

A very similar grouping of isolates was obtained with the neighbor-joining dendrogram of SSR genotypes in Figure 3. Group 1 consisted of 18 of the 19 isolates that clustered with the isolates from durum wheat in Figure 1. The seven isolates in Group 2 included six of the seven PSB isolates in Group 2 in Figure 1. Group 3 isolates included 12 isolates that clustered with the isolates from common wheat, of which nine were found in Group 3 in Figure 1. Isolate Eth 7.2 collected from durum wheat was clustered in Group 1 in Figure 1, but was clustered in Group 3 in Figure 3 for SSR genotype. Isolate PSB 7-3 was clustered in Group 2 in Figure 1, but was clustered in Group 1 in Figure 3. Isolates F58 and F60 that clustered closest to isolates in Group 1 in Figure 1, were clustered in Group 3 in Figure 3 for SSR genotype.

For tests of genetic differentiation, the isolates were placed into three groups that corresponded highly to Groups 1, 2, and 3 in Figures 1 and 3. The eight PSB isolates originally collected from durum wheat with B---- and D---- phenotypes; the five isolates from Spain, France, Ethiopia, and Mexico from durum wheat; and the six ITA isolates with B---- and D---- phenotypes that were characteristic of durum wheat type isolates were placed in one group of isolates (Table 2). The seven PSB isolates collected from durum wheat that had virulence phenotypes characteristic of common wheat isolates were placed in a second group. The eight French isolates from common wheat and the three Italian ITA isolates with F---- phenotypes that had virulence phenotypes and SSR genotypes typical of isolates from common wheat were placed in a third group (Table 2). The three groups of isolates were highly differentiated for virulence phenotype, with an overall $\Phi_{\mathrm{PT}}$ value of $0.574(P<0.001)$ 
The three groups had pair-wise values of $\Phi_{\mathrm{PT}}$ that were all highly significant $(P<$ 0.001 ). The three groups were also highly differentiated for SSR genotypes with an overall $F_{\mathrm{ST}}$ of $0.261(P<0.001)$ and overall $R_{\mathrm{ST}}$ of $0.725(P<0.001)$. The three groups had pair-wise values of $F_{\mathrm{ST}}$ and $R_{\mathrm{ST}}$ that were all highly significant $(P<0.001)$. The seven PSB isolates in the second group with virulence characteristics of common wheat isolates were more closely related to the isolates from durum wheat in the first group $\left(R_{\mathrm{ST}}=0.251\right)$ compared with isolates from common wheat in the third group $\left(R_{\mathrm{ST}}=0.636\right)$. The Mantel coefficient between the virulence distance matrix and the SSR allele distance matrix for all 37 isolates was $0.74(P=1.0)$, which indicated significant correlation between virulence phenotypes and SSR genotypes in this set of $P$. triticina isolates.

\section{DISCUSSION}

Within the two Italian collections (PSB and ITA isolates) of $P$. triticina characterized in this study, there were highly significant differences in SSR genotypes and virulence phenotypes. Eight of the PSB isolates had virulence phenotypes and SSR genotypes characteristic of $P$. triticina from durum wheat. The other seven PSB isolates were somewhat unique because these were virulent to genes $L r 2 c, L r 3 a$, and $\mathrm{Lr} 3 \mathrm{bg}$, similar to isolates from common wheat, but had SSR genotypes that were distinct compared to the isolates from durum wheat and were also distinct compared to the ITA and French isolates from common wheat. Of the nine ITA isolates, six had virulence phenotypes and SSR genotypes characteristic of isolates from durum wheat, and the other three isolates had characteristics of isolates from common wheat. The difference in the $F_{\mathrm{ST}}$ and $R_{\mathrm{ST}}$ values for differentiation of the SSR genotypes reflects the large difference in the molecular weights of the SSR alleles between the three groups of isolates. Since $R_{\mathrm{ST}}$ is based on the single step mutation model that accounts for difference in molecular weights between alleles, this value is likely more reflective of the large evolutionary distance between the three groups of isolates.

The differentiation of $P$. triticina virulence phenotypes collected from durum wheat and common wheat has been previously documented in Spain (15), France (4), Mexico $(7,23)$, South America, and Europe (19). In these studies and in the current study, isolates from durum wheat were highly avirulent to most of the $L r$ resistance genes in the differential set, but all were virulent to $\mathrm{LrIO}$ and $\mathrm{Lrl} 4 \mathrm{~b}$ that are located on chromosomes $1 \mathrm{~A}$ and $7 \mathrm{~B}$ (16), respectively. Most of the isolates from durum wheat were also virulent to Lr23, which originated from the durum cultivar Gaza, and most were virulent to Lr33, also postulated to be derived from durum wheat (16). Isolate Eth 7.2 collected from durum wheat had virulence characteristic of isolates from durum wheat, yet had an SSR genotype more similar to the isolates from common wheat. Ordoñez and Kolmer (19) also noted that isolates from durum wheat in Ethiopia had unique SSR genotypes compared to durum isolates from Europe, Mexico, and South America.

Durum and emmer tetraploid wheat varieties have been cultivated for centuries in Italy and Ethiopia. Isolates of $P$. triticina in these countries would have a long history of selection on durum wheat, which may over time have given rise to variants for

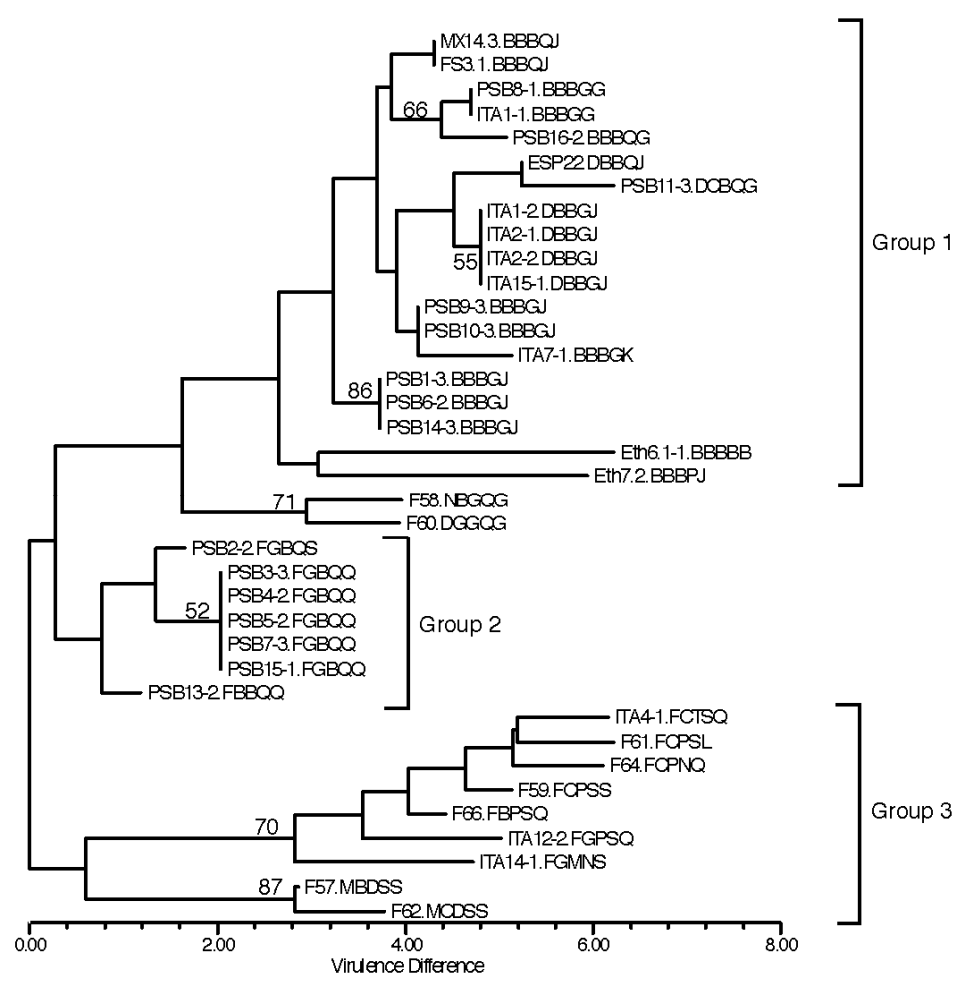

Fig. 1. Neighbor-joining dendrogram based on avirulence/virulence to 22 near-isogenic lines of Thatcher wheat with different leaf rust resistance genes of 37 Puccinia triticina isolates collected from common and durum wheat in Italy, France, Spain, Ethiopia, and Mexico. Bootstrap values $>50$ are indicated for branch points.
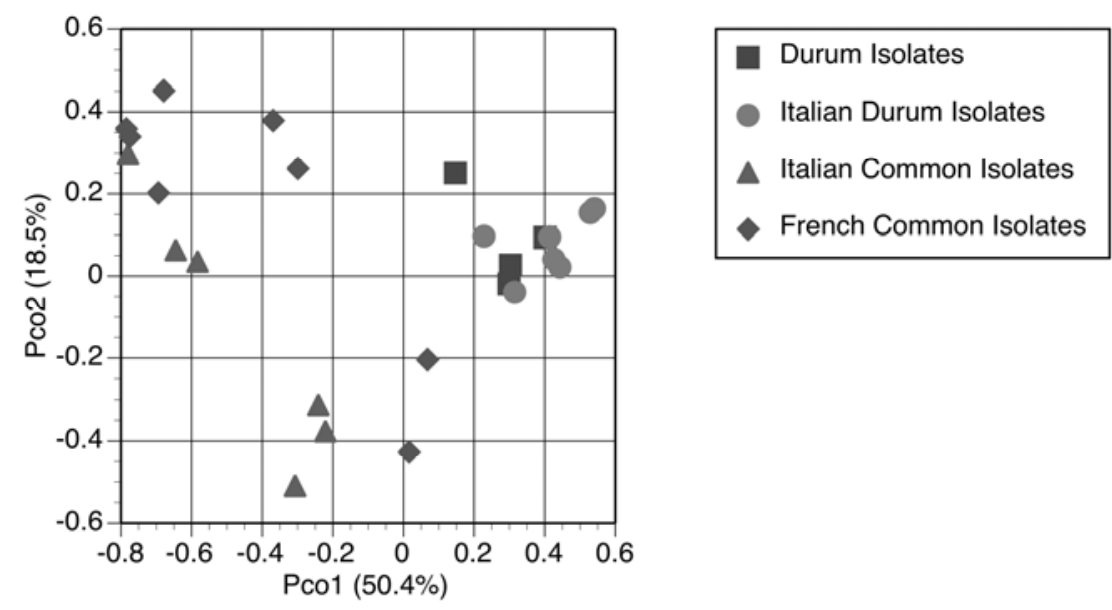

Fig. 2. Principal coordinate analysis plot of virulence phenotypes of 37 Puccinia triticina isolates from common wheat and durum wheat from Italy, France, Spain, Ethiopia, and Mexico based on differences in virulence to Thatcher isogenic lines of wheat with different leaf rust resistance genes. virulence and SSR genotypes that are not present in isolates of $P$. triticina collected from modern durum cultivars in parts of the world where durum cultivation is more recent. This may account for the unique teristic of to a number of $L r$ genes characSSR genotype of isolate Eth 7.2. The seven PSB isolates virulent to $L r 2 c, L r 3, L r 16$, $L r B, L r 10$, and $L r 3 b g$ have a relatively wider virulence spectra to $L r$ genes found in common wheat compared to the other isolates from durum wheat with virulence phenotypes of BBBQJ, BBBGG, DBBQJ, or BBBGJ. 


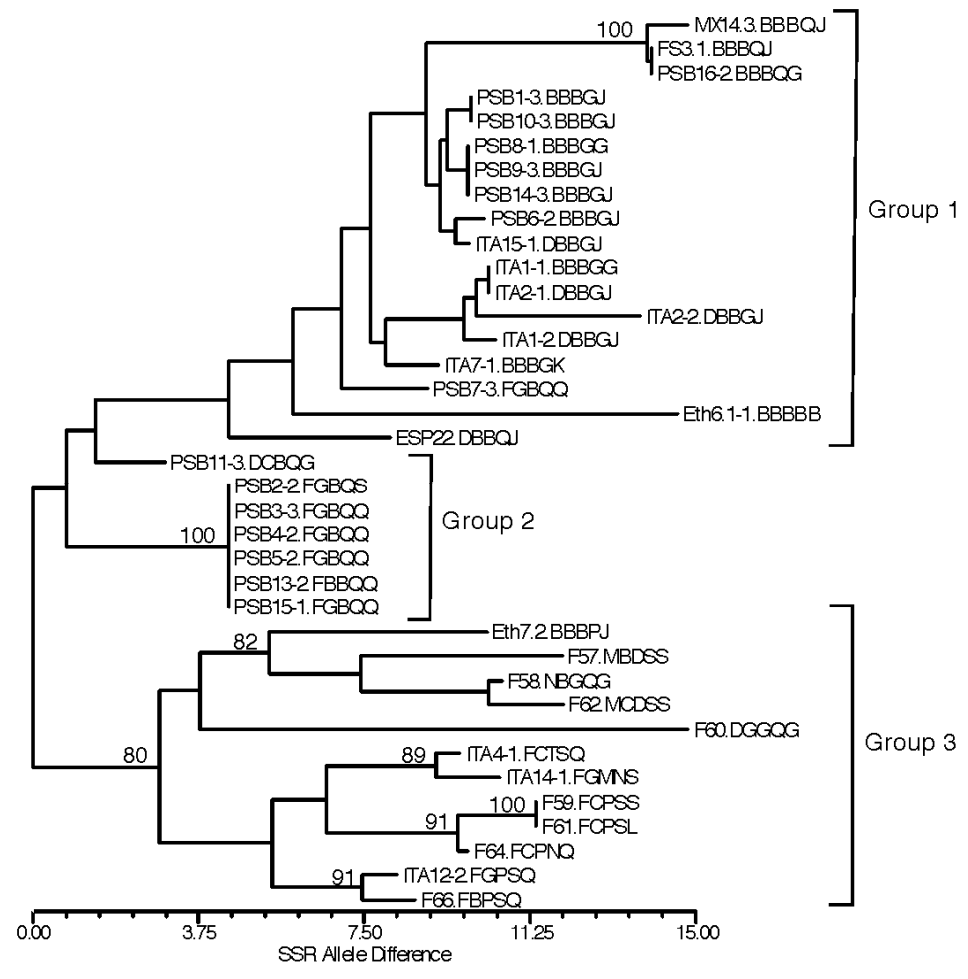

Fig. 3. Neighbor-joining dendrogram based on simple sequence repeat alleles at 15 loci of 37 Puccinia triticina isolates collected from common and durum wheat in Italy, France, Spain, Ethiopia, and Mexico. Bootstrap values $>80$ are indicated for branch points.

The results and interpretation of genetic analyses of leaf rust resistance in durum wheat cultivars depend on the virulence of the P. triticina isolates. Genetic studies of leaf rust resistance in durum wheat cultivars in Canada (26) and the United States (22) used isolates of P. triticina from common wheat. For these studies, it was appropriate to use isolates from common wheat because the P. triticina isolates that are adapted to durum wheats are only rarely found in California and Arizona where durum wheat is grown under irrigation (18), and are not present in the major durum wheat production region in the Great Plains of the United States and Canada. However, in regions where $P$. triticina isolates from common wheat and durum wheat are both present, it would be important to use the appropriate isolate in any genetic analysis of leaf rust resistance in durum wheat. The 15 PSB isolates characterized in this study, although all collected from durum wheat, may differ for virulence to durum wheat cultivars. In an association mapping experiment for leaf rust resistance in durum wheat, four isolates from durum wheat (Eth6.1-1, MX14.3, PSB14-3, and PSB13-2) were used to evaluate a collection of durum accessions from the Mediterranean basin previously characterized for linkage disequilibrium $(13,14)$. The only isolate that was not associated with leaf rust resistance with the durum genotypes was PSB13-2 (13), which suggested that this isolate and the other six PSB isolates with virulence more typical of $P$. triticina from common wheat may not be highly specialized to durum wheat. Since the isogenic Thatcher lines used in this study are common wheat lines, these are not adequate to properly characterize virulence of isolates to durum wheat. To better characterize virulence of $P$. triticina isolates collected from durum wheat, a set of single gene lines of durum wheat that include leaf rust resistance genes derived from different resistance sources will need to be developed.

\section{LITERATURE CITED}

1. Duan, X., Enjalbert, J., Vautrin, D., Solignac, C., and Giraud, T. 2003. Isolation of 12 microsatellite loci, using an enrichment protocol, in the phytopathogenic fungus Puccinia triticina. Mol. Ecol. Notes 3:65-67.

2. Feldman, M. W. 2001. Origin of cultivated wheat. Pages 3-58 in: The World Wheat Book: A History of Wheat Breeding. A. Bonjean and W. Angus, eds. Lavoisier, Paris.

3. Felsenstein, J. 1989. PHYLIP Phylogeny inference package (version 3.2). Cladistics 5:164166.

4. Goyeau, H., Park, R., Schaeffer, B., and Lannou, C. 2006. Distribution of pathotypes with regard to host cultivars in French wheat leaf rust populations. Phytopathology 96:264-273.

5. Herrera-Foessel, S. A., Singh, R. P., HuertaEspino, J., William, J., Rosewarne, G. M., Djurle, A., and Yuen, J. 2007. Identification and mapping of $L r 3$ and a linked leaf rust resistance gene in durum wheat. Crop Sci. 47:1459-1466.

6. Herrera-Foessel, S. A., Singh, R. P., HuertaEspino, J., Yuen, J., and Djurle, A. 2005. New genes for leaf rust resistance in CIMMYT durum wheats. Plant Dis. 89:809-814.

7. Huerta-Espino, J. 1992. Analysis of wheat leaf rust and stem rust virulence on a worldwide basis. Ph.D. thesis. University of Minnesota, St. Paul.
8. Kolmer, J. A., and Liu, J. Q. 2000. Virulence and molecular polymorphism in international collections of the wheat leaf rust fungus Puccinia triticina. Phytopathology 90:427-436.

9. Kolmer, J. A., and Ordoñez, M. E. 2007. Genetic differentiation of Puccinia triticina populations in Central Asia and the Caucasus. Phytopathology 97:1141-1149.

10. Liu, K., and Muse, S. V. 2005. PowerMarker: Integrated analysis environment for genetic marker data. Bioinformatics 21:2128-2129.

11. Long, D. L., and Kolmer, J. A. 1989. A North American system of nomenclature for Puccinia recondita f. sp. tritici. Phytopathology 79:525-529.

12. Maccaferri, M., Mantovani, P., Tubersoa, R., DeAmbrogio, E., Giuliani, S., Demontis, A. Massi, A., and Sanguineti, M. C. 2008. A major QTL for durable leaf rust resistance widely exploited in durum wheat breeding programs maps on the distal region of chromosome arm 7BL. Theor. Appl. Genet. 117:1225-1240.

13. Maccaferri, M., Sanguineti, M. C., Mantovani, P., Demontis, A., Massi, A., Karim Ammar, K. Kolmer, J. A., Czembor, J. H., Ezrati, S., and Tuberosa, R. 2010. Association mapping of leaf rust response in durum wheat. Mol. Breed. DOI: $10.1007 / \mathrm{s} 11032-009-9353-0$.

14. Maccaferri, M., Sanguineti, M. C., Noli, E., and Tubersoa, R. 2005. Population structure and long-range linkage disequilibrium in a durum wheat elite collection. Mol. Breed. 15:271-279.

15. Martinez, F., Sillero, J. C., and Rubiales, D. 2005. Pathogenic specialization of Puccinia triticina in Andulusia from 1998 to 2000. J Phytopathol. 153:344-349.

16. McIntosh, R. A., Yamazaki, Y., Devos, K. M., Dubcovsky, J., Rogers, J., and Appels, R. 2007. Catalogue of Gene Symbols for Wheat. 2007 Supplement. KOMUGI Integrated Wheat Science Database. Available online at http:// www.shigen.nig.ac.jp/wheat/komugi/genes/sym bolClassList.jsp

17. Michalakis, Y., and Excoffier, L. 1996. A generic estimation of population subdivision using distances between alleles with special reference for microsatellite loci. Genetics 142:1061-1064.

18. Ordoñez, M. E., and Kolmer, J. A. 2007. Simple sequence repeat diversity of a worldwide collection of Puccinia triticina from durum wheat. Phytopathology 97:574-583.

19. Ordoñez, M. E., and Kolmer, J. A. 2007. Virulence phenotypes of a worldwide collection of Puccinia triicina from durum wheat. Phytopathology 97:344-351.

20. Payne, T. S., Wanjema, J. K., and Girma, B 2001. Eastern Africa wheat pool. Pages 901938 in: The World Wheat Book: A History of Wheat Breeding. A. Bonjean and W. Angus, eds. Lavoisier, Paris.

21. Peakall, R., and Smouse, P. E. 2006. GENALEX 6: Genetic analysis in Excel. Population genetic software for teaching and research. Mol. Ecol. Notes 6:288-295.

22. Rashid, G., Quick, J. S., and Statler, G. D. 1976. Inheritance of leaf rust resistance in three durum wheats. Crop Sci. 16:294-296.

23. Singh, R. P., Huerta-Espino, J., Pfeiffer, W. and Figueroa-Lopez, P. 2004. Occurrence and impact of a new leaf rust race on durum wheat in northwestern Mexico from 2001 to 2003 Plant Dis. 88:703-708.

24. Slatkin, M. 1995. A measure of population subdivision based on microsatellite allele frequencies. Genetics 159:457-462.

25. Szabo, L. S., and Kolmer, J. A. 2007. Development of simple sequence repeat markers for the plant pathogenic rust fungus Puccinia triticina. Mol. Ecol. Notes 7:708-710.

26. Zhang, H., and Knott, D. R. 1993. Inheritance of adult plant resistance to leaf rust in six durum wheat cultivars. Crop Sci. 33:694-697. 